\title{
Gene Deletions due to Ring Chromosome 16 are Associated with Low Reproductive Potential, a Novel Observation
}

\author{
Tal Lazer and Shevach Friedler* \\ Infertility and IVF Unit, Barzilai University Medical Center, Faculty of Health Sciences, Ben-Gurion University of the \\ Negev, Israel
}

\begin{abstract}
Introduction: Diminished ovarian reserve (DOR) is characterized by decreased number of oocytes associated with poor ovarian response (POR) to controlled ovarian hyperstimulation ( $\mathrm{COH})$. Presently, few specific known chromosomal abnormalities are associated with DOR. Ring chromosome is among the least likely genetic abnormality reported to impact female infertility. It may occurs when two breaks are created in one chromosome and the resulting ends fuse to form a ring, leading to loss of distal genetic material.
\end{abstract}

Case presentation: We reported for the first time on a clinical case of DOR who failed to conceive after repeated IVF treatments and presented with a ring chromosome 16 - 46 XX (r16) with an otherwise normal phenotype.

Conclusion: DOR affects approximately $10 \%$ of women seeking fertility treatment. It is associated with POR to $\mathrm{COH}$, and may be a transient state leading to $\mathrm{POI}$ in a matter of time. It seems that the ring structure itself or genes deletions on ring chromosome 16 may play an important role in the determination of the reproductive potential.

\section{Keywords}

Diminished ovarian reserve, Infertility, Ring chromosome

\section{Introduction}

Chromosomal abnormalities have been long known to affect fertility among females [1]. Presently, few specific known abnormalities are associated with 1 DOR, probably some of these have been recognized as a frequent cause of premature ovarian insufficiency (POI), with a currently estimated prevalence of $10-13 \%$ [2-6]. Genes are known to participate in bipotential gonad formation, sex determination, meiosis, folliculogenesis and ovulation $[2,5]$. Some of them carry the potential to interfere with the efficacy of those processes and contribute to depletion of the ovarian reserve and diminish reproductive success.

Ring chromosome is among the least likely genetic abnormality reported to impact female infertility [7]. It may occur when two breaks are created in one chromosome and the resulting ends fuse to form a ring, leading to loss of distal genetic material [7-9]. The causes of these DNA breaks and the mechanism behind the fusion of the ends are not completely understood. It is considered as a rare condition and typically occurs as de novo event, although cases with familial transmission are also described [7-9]. Most individuals with ring chromosome will present a phenotype associated with the specific affected chromosome which may include dysmorphic features and intellectual disability $[10,11]$ and therefore probably are less likely to present for fertility treatment.
Hereby we report on a rare case of an infertile female diagnosed with a ring chromosome 16, 46 XX (r16), with an otherwise normal phenotype who suffered from DOR and infertility. The specific deleted genes which were diagnosed were not previously reported to be linked to depletion of ovarian reserve.

\section{Case Presentation}

The patient is a 36-years-old female, married for the second time, which was referred to our clinic due to primary infertility for the last 8 years. She is a healthy female, diagnosed with Thalassemia-alpha minor, with no other chronic diseases or operations in her past history. Her family history was negative for chronic or genetic diseases. She had menarche at the age of 12 , with regular menstruation of $28 / 5$ days, since then. Her physical examination included a gynecological examina-

*Corresponding author: Shevach Friedler, Infertility and IVF Unit, Barzilai University Medical Center, Faculty of Health Sciences, Ben-Gurion University of the Negev, Ashkelon, 89123, Israel, Tel: 972537678882

Accepted: May 16, 2019

Published online: May 18, 2019

Citation: Lazer T, Friedler S (2019) Gene Deletions due to Ring Chromosome 16 are Associated with Low Reproductive Potential, a Novel Observation. Annals Gynecol Obstet 3(1):39-42 
tion and gynecological ultrasound revealed a normal female phenotype. Her height $-153 \mathrm{~cm}$, weight - $50 \mathrm{Kg}$ with a BMI of 21.4. Her day 3 baseline hormonal profile was FSH - $11.2 \mathrm{IU} / \mathrm{L}$, LH - $5.2 \mathrm{lU} / \mathrm{L}, \mathrm{E} 2-60 \mathrm{pg} / \mathrm{ml}$. Prolactin, TSH and androgens were within normal limits. Routine pre-gestational genetic mutations screening ruled out fragile-x syndrome.

During her first marriage she underwent 6 cycles of In Vitro Fertilization and embryo transfer (IVF-ET) due to unexplained infertility. During those cycles she demonstrated very poor response to $\mathrm{COH}$. Further evaluation revealed a low anti mullerian hormone level $(\mathrm{AMH})$ of 0.33 . Her karyotype demonstrated a $46 \mathrm{XX}$ with a ring chromosome at one of the 2 chromosomes 16 - 46 XX (r16). Karyotype of both her parents was found normal. She refused to perform a Comparative Genomic Hybridization Assay (CGA) or plan for Preimplantation Genetic Diagnosis (PGD) of the embryos and underwent a $6^{\text {th }}$ IVF-ET cycle. During this cycle, two out of the four oocytes retrieved were fertilized and the transfer of two day 3 embryos resulted in a chemical pregnancy. She got divorced but later at the age of 35 she got married again. At that time her working diagnosis was DOR combined with male factor (MF) due to mild astheno-teratospermia. She underwent additional three IVF cycles with poor ovarian response that failed to achieve any pregnancy. At this point a Chromosomal Microarray Analysis (CMA) analysis was done and revealed deletion at the tip of the short arm of chromosome $16,16 \mathrm{p} 13.3$ of $387 \mathrm{~KB}$ including the genes HBA2, HBA1, AXIN1. In addition, there were deletion areas at the tip of the long arm of chromosome 16,16q24.3 of $368 \mathrm{~KB}$ including the genes MC1R, TUBB3, GAS8. Presently, none of these genes is known to be associated with DOR or infertility.

\section{Conclusion}

Female fertility potential is linked to ovarian reserve and oocyte quality. These parameters may be influenced by genes that participate in key biological processes in the ovary, such as meiosis, follicular development, granulosa cell differentiation/proliferation and ovulation [2,3,12]. DOR, with an imprecise definition of a decreased number or quality of oocytes, affects approximately $10 \%$ of women seeking fertility treatment [13]. It is associated with POR to $\mathrm{COH}$, and may be a transient state leading to $\mathrm{POI}$ in a matter of time. Despite of being investigated widely, precise definition, diagnosis and optimal management of DOR remain an unsolved enigma, with no single most beneficial intervention having been proposed [13]. It might be that DOR in an early age is a pathology, predicting $\mathrm{POI}$ in the future. Recently substantial evidences suggest a genetic basis for POI; studies reported over 60 different genes implicated in the condition [2-4]. Unfortunately, despite this large number of suggested POI related genes, the majority of patients remain without a genetic diagnosis.

Ring chromosome is a cytogenetic anomaly frequently associated with loss of genetic material. Deletions in various genes or loss of distal genetic material at the chromosome as in the formation of ring chromosome may affect different steps of ovarian pool development and expression such as bipotential gonad formation, sex determination, meiosis, follic- ulogenesis and ovulation. As a result, patients who carry such a condition may demonstrate DOR or even POI. The causes of the DNA breaks and the mechanism behind the fusion of the ends are not completely understood. It may involve attraction between the non-homologous end-joining chromosome arms [1] or due to telomere dysfunction, triggering fusion of reactive unstable chromosome ends without major loss of genetic material $[1,7,8,9,11]$. Most cases are sporadic following genetic damage by mutagens like radiation [14]. Rarely, it may be inherited, ending in supernumerary condition.

Ring chromosome 16 is a rare event and the data in the literature is very limited [7-9]. Most cases described presented with high degree of phenotype variability with the majority of cases demonstrating multiple malformations. For our knowledge, this case is the first ever reported ring chromosome 16 with normal phenotype and DOR presenting with deletion areas on both arms. The size and the genes involved were evaluated and specified. Genes that were deleted at the tip of the short arm of chromosome 16 in our patient and so far were not associated with DOR or POI are AXIN, HBA1 and HBA2.

AXIN - a gene located on the short arm of chromosome 16- 16p13.3.

The gene encodes a G-protein signaling (RGS) domain regulatory cytoplasmic protein and axin (DIX) domain. It has regulatory roles in the 1 WNT-beta-catenin signaling pathway during embryonic development and tissue homeostasis in adults. This pathway modulates cellular differentiation, proliferation, movement, and polarity $[15,16]$. Mutations in AXIN1 is related to caudal duplication anomaly (CADUA): a condition characterized by the occurrence of duplications of different organs in the caudal region. In addition, mutations in AXIN1 are also involved in hepatocellular carcinoma (HCC). Mutations in this gene have also been associated with hepatoblastomas, ovarian endometriod adenocarcinomas, and medullablastomas [17].

The HBA1 and HBA2 are two, nearly identical, alphaglobin genes, located closely, at the alpha-globin locus of chromosome 16. Deletions of one or both genes are the most common cause of alpha thalassemia. People with alpha thalassemia trait may have unusually small, pale red blood cells and mild anemia $[18,19]$. These deletions may be associated with the patients' thalassemia.

Genes that were deleted at the tip of the long arm of chromosome 16 were MC1R, TUBB3 and GAS8.

MC1R: A gene located on the long arm of chromosome $16 q 24.3$, with an autosomal recessive mode of inheritance. The melanocortin 1 receptor (MC1-R) is part of the melanocortin system, consists of melanocyte-stimulating hormones ( $\alpha, \beta$ and $\gamma-\mathrm{MSH})$ and their cognate receptors. It regulates a variety of physiological functions, ranging from skin pigmentation to centrally mediated energy balance control. At the cellular level, the biological actions are mediated by a family of five G-protein coupled melanocortin receptors, named from MC1-R to MC5-R, which all have distinct tissue distribution and functional properties. MC1-R 
was the first receptor member to be cloned and is known to be an integral regulator of skin pigmentation. Over the past years it has become evident that MC1-R signaling exerts immunomodulatory effects in the cells of the immune system like monocytes and macrophages. It was found to exert antiinflammatory actions upon stimulation with its natural ligand $\alpha$ - melanocyte-stimulating hormone ( $\alpha-\mathrm{MSH})[20]$.

TUBB3: A gene located on the long arm of chromosome $16 q 24.3$, with an autosomal dominant mode of inheritance.

TUBB3 belongs to a group of genes encoding for several different neuronal a- and b-tubulin isotypes forming tubulin heterodimers in the nervous system. These dynamic polymeric structures that are continuously incorporated and released, function in mitosis, intracellular transport, neuron morphology, and ciliary and flagellar motility.

TUBB3 mutations cause a spectrum of brain malformations and neurological symptoms, and specific missense mutations correlate to certain phenotypes. A subset of patients has congenital fibrosis of the external ocular muscles (CFEOM type 3) due to hypoplasia of the oculomotor nerves [21].

GAS8: The gene is located on the long arm of chromosome 16 q24.3 with an autosomal recessive mode of inheritance. The GAS8 gene encodes for the protein DRC4, a component of the nexin-dynein regulatory complex (N-DRC) that plays an important structural and functional role in ciliary movement. Mutation in the gene presents a phenotype of primary ciliary dyskinesia. Loss of heterozygosity involving chromosome $16 q 24.3$ is common in breast and prostate cancer and suggests the presence of a tumor suppressor gene [22].

Presently, none of these genes is known to be associated with infertility or any form of DOR. The deletion of one of the genes HBA2 or HBA1 may be associated with the patients' thalassemia. Even though mutation in the gene TUBB3 may be associated with a variety of abnormalities as brain anomalies and congenital fibrosis of extraocular muscles none of these were expressed in our patient.

DOR may be a transient state leading to POI in a matter of time. Elucidating the genetic and molecular basis in women with DOR who may deteriorate to POI is of importance in understanding ovarian physiology and better understanding disease mechanisms [23-25]. This will ultimately aid the development of better treatments and providing a better genetic counseling and fertility guidance $[26,27]$. Women demonstrating DOR in an early age, before presenting certain perturbations of POI can be offered the option of oocyte cryopreservation, with later thawing and use in assisted reproductive technology. Once additional variants are detected, it might become possible to predict DOR and later fore see the age of premature menopause in women at risk for POI [28].

In conclusion, we report here the deletion of several genes diagnosed in a patient with ring 16 mutations who suffers from DOR and infertility. These genes have not been previously reported in association with depletion of ovarian reserve or infertility. It seems that the ring structure itself or one or more gene deletions on ring chromosome 16 may play an important role in the determination of the reproductive potential.

\section{Acknowledgement}

None.

\section{Statement of Ethics}

The subject have given her informed consent to publish the case.

\section{Disclosure Statement}

The authors have no conflicts of interest to declare.

\section{Funding Sources}

None declared.

\section{References}

1. Guilherme R, Klein E, Hamid A, et al. (2013) Human ring chromosomes - New insights for their clinical significance. Balkan J Med Genet 16: 13-20.

2. Rossetti R, Ferrari I, Bonomi M, et al. (2017) Genetics of primary ovarian insuffiency. Clin Genet 91: 183-198.

3. Tucker EJ, Grover SR, Bachelot A, et al. (2016) Premature ovarian insufficiency: New perspectives on genetic cause and phenotypic spectrum. Endocr Rev 37: 609-635.

4. Jiao X, Ke H, Qin Y, et al. (2018) Molecular genetics of premature ovarian insufficiency. Trends Endocrinol Metab 29: 795-807.

5. Qin Y, Jiao X, Simpson JL, et al. (2015) Genetics of primary ovarian insufficiency: New developments and opportunities. Hum Reprod Update 21: 787-808.

6. Laven JS (2016) Primary Ovarian Insufficiency. Semin Reprod Med 34: 230-234.

7. Morin SJ, Eccles J, Iturriaga A, et al. (2017) Translocations, inversions and other chromosome rearrangements. Fertil Steril 107: 19-26.

8. Conte RA, Kleyman SM, Kharode C, et al. (1997) Delineation of a ring chromosome 16 by the FISH-technique: A case report with review. Clin Genet 51: 196-199.

9. Cignini P, Dinatale A, D'Emidio L, et al. (2011) Prenatal diagnosis of a fetus with de novo Supernumerary ring chromosome 16 characterized by array comparative genomic hybridization. AJP Rep 1: 29-32.

10. Chodirker BN, Ray M, McAlpine PJ, et al. (1988) Developmental delay, short stature and minor facial anomalies in a child with ring chromosome 16. Am J Med Genet 31: 145-151.

11. He W, Tuck-Muller CM, Martinez JE, et al. (2002) Molecular characterization of a ring chromosome 16 from a patient with bilateral cataracts. Am J Med Genet 107: 12-17.

12. Patiño LC, Beau I, Carlosama C, et al. (2017) New mutations in non-syndromic primary ovarian insufficiency patients identified via whole-exome sequencing. Hum Reprod 32: 1512-1520.

13. Huang Y, Li J, Zhang F, et al. (2018) Factors affecting the live-birth rate in women with diminished ovarian reserve undergoing IVFET. Arch Gynecol Obstet 298: 1017-1027.

14. Jacobs PA, Frackiewicz A, Law P, et al. (1975) The effect of structural aberrations of the chromosomes on reproductive fitness in man. II. Results. Clin Genet 8: 169-178. 
15. $\mathrm{OMIM}^{\circ}$ Online Mendelian Inheritance in $\mathrm{Man}^{\circ}$, An Online Catalog of Human Genes and Genetic Disorders, AXIS INHIBITOR 1, AXIN1.

16. NCIB, Gene ID: 8312.

17. AXIN1 gene, NIH, Genetics home reference.

18. HBA1 gene, NIH, Genetics home reference.

19. OMIM Online Mendelian Inheritance in Man', An Online Catalog of Human Genes and Genetic Disorders, HBA1 and the HBA2.

20. OMIM ${ }^{\circ}$ Online Mendelian Inheritance in Man', An Online Catalog of Human Genes and Genetic Disorders, MC1R.

21. $\mathrm{OMIM}^{\bullet}$ Online Mendelian Inheritance in Man', An Online Catalog of Human Genes and Genetic Disorders, TUBB3.

22. $\mathrm{OMIM}^{\circ}$ Online Mendelian Inheritance in Man ${ }^{\circ}$, An Online Catalog of Human Genes and Genetic Disorders, GAS8.
23. Persani L, Rossetti R, Cacciatore C, et al. (2011) Genetic defects of ovarian TGF- $\beta$-like factors and premature ovarian failure. J Endocrinol Invest 34: 244-251.

24. Laissue P, Vinci G, Veitia RA, et al. (2008) Recent advances in the study of genes involved in non-syndromic premature ovarian failure. Mol Cell Endocrinol 282: 101-111.

25. Persani L, Rossetti R, Cacciatore $C$ (2010) Genes involved in human premature ovarian failure. J Mol Endocrinol 45: 257-279.

26. Fortuno C, Labarta E (2014) Genetics of primary ovarian insuffiency: A review. J Assist Reprod Genet 1: 1573-1585.

27. Jin $M, Y u Y$, Huang $H(2012)$ An update on primary ovarian insuffiency. Sci China Life Sci 55: 677-686.

28. Desai S, Rajkovic A (2017) Genetics of reproductive aging from gonadal dysgenesis through Menopause. Semin Reprod Med 35: 147-159. 